\title{
Reflexiones en torno a la emergencia del feminismo mapuche en la comuna de Pudahuel
}

Karina Ahumada Pailahueque *

\begin{abstract}
Resumen
El presente artículo se pregunta por las posibilidades y obstáculos para la emergencia de un "feminismo mapuche" en la comuna de Pudahuel. Para ello, recurro a un ejercicio reflexivo basado en mi experiencia laboral, con nueve organizaciones indígenas del territorio, durante el transcurso de dos años y medio (2016-2019). La hipótesis del texto es que la dualidad, la unidad de lucha, la violencia vivida al interior y fuera del hogar, la invisibilización por parte de los feminismos "blancos" y el llamado a representar la dignidad de un pueblo, son algunas de las paradojas que las mujeres mapuche compartimos en el proceso de construcción de una subjetividad política feminista.
\end{abstract}

Fecha de recepción: Marzo 2019

Fecha de aprobación: Junio 2019

Palabras clave: dualidad, unidad de lucha, violencia de género, feminismo mapuche

\section{Introducción}

Para comenzar, me pregunto si el "feminismo mapuche" existe como tal, en Chile. La pregunta cobra relevancia porque dicho feminismo es a menudo cuestionado, tanto por las propias mujeres mapuche, como por el feminismo occidental. Aunque la respuesta es aparentemente positiva, y por otra parte es posible decir que existen diversos feminismos indígenas, hay varias mujeres de pueblos originarios que trabajan por los derechos de las mujeres de su comunidad u organización, pero no se reconocen como feministas. Dicho esto, la cantidad de mujeres mapuche que despliegan una acción feminista en el territorio y las diversas investigaciones sobre el movimiento, me despejan las dudas sobre su existencia (Arellano 2015; Forciniti y Palumbo 2012; Gargallo 2011; Hernández 2008; Lorente 2005; Lugones 2010; Quiñimil2012; Rivera 1999, entre otros estudios).

\footnotetext{
Socióloga. Universidad Academia de Humanismo Cristiano. Magíster en Estudios de Género y Cultura, mención Ciencias Sociales. Universidad de Chile. Email: kv.ahumada2@gmail.cl
} 
Sin embargo, me interesa situar el feminismo mapuche en relación con las posibilidades y obstáculos para su emergencia en el medio urbano local, en este caso la comuna de Pudahuel. Para aquello, voy a recurrir a un ejercicio reflexivo basado en mi experiencia de trabajo con nueve organizaciones mapuche del sector, desde el año 2016 al presente.

Me parece relevante además especificar desde qué lugar "hablo y escribo yo", cual es mi posición: aunque tengo estudios profesionales en sociología y especialidad en los temas de género y cultura, declarándome abiertamente feminista, estoy en pleno proceso de búsqueda de mi identidad como mujer mapuche, producto del despojo de la historia oficial y de mis raíces ancestrales, reconociéndome una aprendiz de su cultura y cosmovisión. En ese sentido, mi posicionamiento responde al tránsito de este texto, que se pregunta por categorías como la dualidad, la unidad de lucha, la violencia vivida al interior y fuera del hogar, la invisibilización por parte de los feminismos "blancos" y el llamado a representar la dignidad de un pueblo, que a mi entender son algunas de las paradojas que las mujeres mapuche compartimos en el proceso de construcción de una subjetividad política feminista.

\section{Antecedentes}

Pudahuel del mapuzungun significa "lugar de pantanos"1. Es una comuna ubicada en el sector norponiente de la capital. Limita al Norte con la comuna de Lampa, al sur con Maipú, al este con Quilicura, Renca, Cerro Navia, Lo Prado y Estación Central, y al oeste con Curacaví. Fue fundada en 1897 y hasta 1981 fue llamada "Barrancas", y se caracterizaba por un paisaje rural, fruto de las migraciones de campesinos/as durante el siglo XX, y posteriormente, con las transformaciones urbanas de los años sesenta, se consolida dentro del Gran Santiago. Sin embargo, con la dictadura militar se acentúan inconvenientes tanto en la ordenación como en la re-organización de la comuna ${ }^{2}$, dividiéndose en tres zonas: Pudahuel norte, sur y rural, que se mantienen en la actualidad.

Según Reportes Estadísticos Comunales, en el año 2017 la comuna registra una población de 230.293 habitantes, de los que un 11,75 \% declara pertenecer al pueblo mapuche. También los Censos de Población y Vivienda 2002-2017, sumado a la Encuesta Casen del año 2015, sustentan esta cifra.

A mediados del año 2016 llego a trabajar al municipio como Coordinadora del Programa Asuntos Indígenas ${ }^{3}$, perteneciente a la Dirección de Desarrollo Comunitario, cuyo objetivo principal busca "Contribuir al desarrollo integral y a la participación de la población indígena de la comuna, a través del trabajo conjunto con la Mesa de Pueblos Originarios, cuyas acciones apuntan a la difusión y promoción de la cultura y tradiciones indígenas..." (Plan Operativo, 2018).

Es necesario señalar que a diferencia de otros programas, la Mesa de Pueblos Originarios compuesta por nueve organizaciones mapuche (mixtas) denominadas: Consejo Mapuche de Pudahuel, Meli Rewe, Witrunko Mapu, Rayenco, Club del Adulto Mayor Kosquilla Rayen, Comité de Allegados Willimapu ${ }^{4}$, Grupo de Educación Intercultural, Comité de Allegados

\footnotetext{
${ }^{1}$ Aproximación de María Huenuñir Antihuala, poeta y escritora mapuche, oriunda de Cayumapu Alto, Panguipulli, trabaja como Educadora de Lengua v Cultura Indígena $(\mathrm{EICl})$ en jardines infantiles y escuelas de la región Metropolitana, reside en Pudahuel.

2 Para mayor información revisar tesis "Recuperación del rol de las mujeres pobladoras en la historia de Pudahuel (1965-1989): Luchas y sueños por extrapolar". Ahumada, 2016.

${ }^{3}$ Anualmente el Programa cambia de nombre, por dictamen de la Contraloría.

${ }^{4}$ Cabe mencionar que la participación de la organización en la Mesa de Pueblos Originarios, se ha visto debilitada el último tiempo.
} 
Kallfu Rayen, Agrupación Cultural Peumayain, es fundamental. Dado que se diseña, planifica y evalúa mediante consulta, tal como lo establece el Convenio 169 de la OIT, que protege los derechos de los pueblos indígenas y tribales, garantizando su participación en la toma de decisiones.

\section{Dualidad o binarismo jerárquico}

Una primera reflexión desde la cual quisiera partir, dice relación con un aspecto de la cosmovisión mapuche: la dualidad. Aún recuerdo cuando en el "Taller de Lengua y Cultura Mapuche", la monitora aludía a la visión dual del mundo: tierra/cielo; sol/luna; lluvia/sequía; hombre/mujer, etc. Donde los dos polos existen, pero son complementarios o bien constituyen una dualidad en equilibrio.

Dicho principio contempla unidad, respeto y reciprocidad con la ñukemapu (madre tierra) y la naturaleza. De ahí cierto distanciamiento con la categoría "género", que significaría de acuerdo a esta mirada, alterar el equilibrio en las relaciones entre mujeres y hombres, generando un antagonismo jerárquico.

Al respecto, me surgieron algunas preguntas, tales como ¿en la cosmovisión mapuche se asume como "natural" el equilibrio en las relaciones entre mujeres y hombres?, también ¿las/los integrantes de las organizaciones de la comuna se guían por estos valores en la cotidianeidad? dado que tales elementos han sido trastocados y permeados por la estructura patriarcal de las sociedades dominantes, más aún cuando en Chile el actual modelo económico promueve la competencia, el individualismo y el exitismo.

Sin embargo, me detuve un momento y pensé que este cuestionamiento hacia la dualidad, responde a una visión occidental ligada al binarismo, donde un término suplementa y no complementa a otro, es decir, cuando uno de esos términos se vuelve "universal" y el otro termina siendo "resto". Tal como señalan Segato (2011) y Gargallo (2012), el binarismo entre lo femenino y lo masculino, introducido por la colonización, fue adoptado y aprovechado por los hombres indígenas para acceder a puestos de poder político 0 económico.

Por otra parte, aparece una suerte de idealización del pasado y negación defensiva del presente, sugerida por Ancán (2010) y Menard (2009) respecto a la distinción de una cultura mapuche "real" y otra "actual", corrompida. Siguiendo sus argumentos, es importante dilucidar cuál es el trasfondoque está operando en la representación sobre ese pasado armónico entre mujeres y hombres. Invirtiendo las valoraciones de una dicotomía históricamente colonialista (puro/impuro), la noción de complementariedad arriesga caer en una trampa, ya señalada por Gargallo (2011 y 2012): se convirtió en una "obligación" de servicios por parte de las mujeres hacia los hombres indígenas, cubriéndose con discursos de hábitos y de costumbres ancestrales.

Junto con ello, sospecho de cierta dosis heteronormada, en tanto la complementariedad podría reforzar una representación hegemónica de "mujer mapuche legítima" que custodia la cosmovisión de su pueblo, invisibilizando la existencia de otras identidades y subjetividades mapuche.

\section{Frente de lucha conjuntos}

Un segundo aspecto que me suscita interéses el discurso de la "unidad de lucha" contra la discriminación y el machismo, al interior de las organizaciones. La lucha esasumida colectivamente, ya que contempla tanto a los hombres como a las mujeres. No se trata de una lucha contra la dominación masculina, sino que es conjunta para restablecer comunidad en base a ladualidad complementaria y justa. Por lo que, la primera disputa es por los 
derechos colectivos, entre ellos, el principal, el derecho al territorio en el cual se asienta la comunidad.

Es frecuente escuchar de parte de pulamngen (hermanas/hermanos), que el feminismo no encaja con la realidad mapuche, ya que plantea una lucha separatista, en algunos casos anti-hombres, debilitando la unidad del movimiento. Me pregunto entonces ¿Es la lucha por los derechos colectivos un motivo para distanciarse del feminismo? ¿en qué medida la "unidad en la lucha"afecta en la autodefinición de las mujeres mapuche como feministas?

Es plausible pensar que un pueblo excluido de la esfera política ${ }^{5}$ ha generado puntos de vista en común con el pensamiento feminista; especialmente con el que caracterizó al periodo antidictatorial, en términos de interpelar al Estado, escenario en el que hasta hoy se han centrado las demandas del pueblo mapuche.

El hecho de que algunas mujeres mapuche les cueste identificarse como feministas puede deberse a las posiciones y situaciones de subordinación ${ }^{6}$ en que se encuentran en la sociedad chilena. Ello provoca efectos de aglutinarse, antes que de separarse de los hombres para formar un movimiento propio de mujeres. Quizás sea está una razón por la que en Pudahuel sólo hay organizaciones mixtas, aun cuando han contribuido a que se definan como mujeres mapuche.

No obstante lo anterior, se deja entrever la necesidad y la exigencia de pulamngen (hermanas) hacia las organizaciones, por ser más valoradas, escuchadas e incidir en la toma de decisiones. La lucha por demandas colectivas (pueblo) y la lucha por demandas individuales (género), al parecer deben complementarse, ninguna en desmedro de otra, lo que sin lugar a dudas es un desafío a enfrentar.

\section{Violencia contra las mujeres mapuche}

Un tercer aspecto que me parece interesante de reflexionar es la violencia hacia las mujeres mapuche a nivel local. Dado que en el quehacer con las organizaciones de la comuna, poco se habla y en algunos casos se oculta. Me llama la atención que sólo las mujeres, sean quienes la posicionan y politizan.

Según Painemal (2013), el abordaje de la violencia intrafamiliar y otros problemas específicos como la salud sexual y reproductiva, motivaron el surgimiento de organizaciones propiamente femeninas en la década de los años noventa, a nivel urbano como rural, las que no estuvieron exentas de conflictos con sus pares masculinos, quienes las acusaron de estar dividiendo el movimiento mapuche y de estar siendo influenciadas por corrientes feministas. A pesar de ello, las mujeres continuaron trabajando en sus organizaciones, fortaleciendo su autoestima y visibilizando sus liderazgos.

El hablar con pulamngen que están en situación de violencia, al interior de sus hogares, no es fácil, debido al miedo que les ocasiona denunciar a sus agresores y la respuesta machista y patriarcal obtenida por parte del Estado chileno, que en vez de afrontar el problema, tiende a estigmatizar al pueblo mapuche ${ }^{7}$. Son incontables las veces que he tenido que escuchar "el hombre mapuche es muy machista, alcohólicoy violento",

\footnotetext{
${ }^{5}$ En términos de reconocimiento y de representatividad.

${ }^{6}$ Me refiero a los cruces entre clase, etnia, edad, orientación sexual, entre otros.Tales aspectos, no habrían sido considerados por la política feminista, la que aislando todas las diferencias y contextos, habría establecido, por los menos en sus inicios, un único sujeto mujer.

7 Un hecho desdeñable, revela la información entregada por Sernam al diario Austral, el 11 de marzo de 2013. Ahí se señala que en casos de violencia contra mujeres mapuche, fue invocada la costumbre ancestral presente en el Convenio 169 de la OIT. Esto generó que los agresores sólo pidieran disculpas públicas dejándoles exentos de penalidad.
} 
"antiguamente un hombre tenía varias mujeres como esposas", "los dirigentes no te van a escuchar, porque eres mujer y más encima joven", entre otros comentarios estereotipados y teñidos de fundamentalismos. Me pregunto si acaso un hombre, dirigente, sin ascendencia indígena, eventualmente sería menos machista que el mapuche. O si es conveniente presuponer que la cultura no tuvo historia, con el argumento de que "siempre fue así y que no va a cambiar".

Poco tiempo pasó para que en untrawün (encuentro, reunión) y de manera inesperada aflorara la forma más usada y eventualmente correcta de proceder ante los casos expuestos de violencia doméstica. Se planteó que dicha situación, debe ser tratada al interior de las organizaciones, considerando el azmapu $u^{8}$, el cual contiene normas de un territorio que rigen a las personas y regulan su interacción con los demás seres vivos. No obstante, surgen algunas preguntas ¿Es posible aplicar el azmapu en Pudahuel? ¿Hay funciones establecidas que deban cumplir las organizaciones para solucionar los conflictos?, entre otras.

De acuerdo a Painemal y Álvarez (2016), un primer elemento que ayuda a esclarecer estas dudas es la existencia de un desequilibrio en las relaciones familiares mapuche provocado por la pérdida de identidad y por el proceso de dominación sociocultural ejercido desde el Estado chileno.

Un segundo elemento que propone Calfio (2009), es que producto de la colonización el papel reproductivo perdió valor social y ya no fue apreciado como en tiempos de libertad. Lamentablemente he podido palpar en algunos testimonios la desvalorización y la falta de espacio propio, donde existe incluso un cuestionamiento hacia "ser mujer" por estar permanentemente recibiendo humillación y menosprecio de hombres acomplejados.

Considerando ambos argumentos, creo que una de las posibles soluciones apunta a comprender y aportar desde lo mapuche, es decir, visualizar como las organizaciones se hacen parte de este problema que está presente y que no pasa sólo por los mecanismos que las instituciones públicas, en este caso el municipio, pueden implementar a través de diferentes programas sociales. En este sentido, es primordial que las mujeres reivindiquemos estratégicamente elementos de la cosmovisión, como la dualidad y la complementariedad. La demanda de principios y valores ancestrales nos permite recuperar el estatus femenino en la comunidad, exigir cambios en la relación con los hombres mapuche, y que se reconozca nuestra participaciónen la lucha del movimiento y/o pueblo.

\section{Invisibilización en los feminismos occidentales y representación de un pueblo}

Un cuarto aspecto a considerar es la invisiblización en los feminismos occidentales y la representación "dignificada" de un pueblo. Para las mujeres mapuche de Pudahuel (y me incluyo), la lucha por la identidad no se puede desprender de la lucha por la tierra y por la propia cultura, pese a que estamos en la ciudad. Para algunos grupos y colectivos feministas, el hecho de que la lucha contra el patriarcado quede en segundo plano, es interpretado como incapacidad nuestra para cuestionar al poder masculino presente en el pueblo mapuche. Sin embargo, me impresiona que muy pocas cuestionen el poder entre mujeres. El racismo de las mujeres blancas y mestizas, a mi entender, está casi tan latente como el patriarcado de los hombres, blancos, mestizos e indígenas.

"En América Latina, en gran medida, las mujeres blancas han tenido con las indígenas y afrodescendientes una relación de matrona-sirvienta, de propietaria-esclava o de señora-

\footnotetext{
${ }^{8}$ Azmapu: conjunto de normas que ordenan todo lo que existe, en correspondencia a la persona mapuche, la naturaleza y el universo. Implica la armonía y el equilibrio a nivel social, político, jurídico, espiritual y cultural.
} 
muchacha. La historia nos ha hecho desiguales y sería muy desafortunado ocultar esas asimetrías bajo un argumento falaz de la universalidad de una forma de ser mujer, levantando una única bandera de liberación" (Cumes, 2009: 34).

Recordemos que una parte no despreciable de los feminismos, se centran en la búsqueda de igualdad y/o equidad, la cual universaliza la lucha sin tomar en cuenta cruzamientos de clase, raza, orientación sexual e identidad con el territorio. Estas formas de organización son percibidas por las mujeres mapuche e indígenas como un feminismo hegemónico, clasista, blanco, y endocéntrico.

"Abrazar este feminismo sin cuestionarlo nos parece peligroso; la colonialidad y el patriarcado no desaparecen por trabajar "entre mujeres" (...). Por lo tanto, es necesario que los movimientos feministas den un paso mayor y que primero hablen y se hagan cargo de sus privilegios..." (Painemal y Cañet, 2018).

Al parecer resulta necesario alejarse de la mirada tradicional feminista, que visualiza la vida de las mujeres indígenas, desde un enfoque euro y norcentrado, nacido de la modernidad y que se ancla en la noción de patriarcado para articular las luchas (Espinoza, 2014). Es más pertinente hablar de un "feminismo localizado" que afirma su identidad en el territorio y en la historia en la que se tiene raíces, alejándonos del paradigma que ubica a los pueblos originarios como "otros" en sus tierras.

También me parece importante destacar que las reivindicaciones de índole étnicas, en su mayoría contienen una supuesta neutralidad de género, que cae en el riesgo de "esencializar" a las culturas indígenas, en tanto se las comprende como valores y costumbres "puras", al margen de las relaciones de poder, y en ese cometido, se utiliza a las mujeres como "símbolos de sus pueblos".

\section{A modo de conclusión}

Como he intentado mostrar en este texto, en Pudahuel las mujeres mapuche organizadas afirman su identidad en el principio de la complementariedad y están intentando articular unalucha contra el patriarcado y el racismo. En relación a un proceso que implica resignificar los valores indígenas dentro de las organizaciones, no solo la categoría ya citada, sino también el equilibrio y la reciprocidad, quedeben estar en sintonía con los cambios en las necesidades y los deseos de las personas. Mi llamado es analizar de manera crítica y profunda su sustento en la práctica, a fin de que no sirva de manto silenciador de opresiones sistemáticas. Así tal vez se convierta en una herramienta para retornar al orden comunitario. La diversidad es una de las características de las mujeres mapuche de Pudahuel: hay quienes se consideran a sí mismas feministas y se reconocen como tales; otras despliegan una acción feminista sin aceptar pertenecer al movimiento: también hay mujeres que no quieren saber nada con el feminismo por considerarlo ajeno y foráneo, yaquellas que trabajan únicamente por los derechos de su organización.

Sin embargo, hay un terreno de lucha común que va más allá de la comuna, que es la lucha contra la globalización neoliberal y el extractivismo, donde la amenaza de la industria forestal y minera es permanente. El reconocimiento como pueblo y la aceptación de las diferencias, son esenciales para el establecimiento de un diálogo fecundo previo a las alianzas con los movimientos de mujeres, para construir un feminismo mapuche y una sociedad más justa. 
Creo que en este sentido, es muy importante elaborar de manera colectiva el trauma que se repite y transmite de forma intergeneracional, hay que reconocer quehemos experimentadodistintas formas de violencia y al mismo tiempo ser capaces de rescatar y potenciar resistencias milenarias, que provienen de nuestros Kuifikecheyem (ancestros/as).

Mi intención no es llamar a la creación de organizaciones indígenas más inclusivas y/o masivas, sino a des-centrar al feminismo blanco/liberal. El asunto requiere abordar el punto de vista histórico que comienza con el colonialismo y el despojo, y que necesariamente nos insta a desmantelar las jerarquías, inclusive las que imponen las teorías decoloniales y grupos feministas.

\section{Bibliografía}

Ahumada, Karina (2016): "Recuperación del rol de las mujeres pobladoras en la historia de Pudahuel (1965-1989): Luchas y sueños por extrapolar”. Tesis (Magíster en Estudios de Género y Cultura). Santiago de Chile: Universidad de Chile, Facultad de Ciencias Sociales.

Ancán, José (2010): "Negritud y "cosmovisionismo" mapuche frente al poder (neo) colonial. Apuntes (muy) preliminares para una reflexión (auto) crítica", en Oliva, Stecher y Zapata (eds.):Aimé Césaire desde América Latina. Diálogos con el poeta de la negritud, p.101-126. Santiago de Chile: Ediciones Facultad de Filosofía y Humanidades, Universidad de Chile.

Arellano, Claudia (2015): "Despatriarcalizando: Julieta Paredes y su vinculación con el discurso político y poético de mujeres mapuche",en Revista Antropologías del Sur, Vol. 2, No 4, p. 53-65.

BCN (2017): Reportes Estadísticos Comunales. Pudahuel. Disponible en: https://reportescomunales.bcn.cl/2017/index.php/Pudahuel

Calfio, Margarita (2009): "Mujeres mapuche, voces y acciones en momentos de la historia", en Pequeño (comp.): Participación y políticas de mujeres indígenas en contextos latinoamericanos recientes, p.91-109. Quito, Ecuador: Ediciones FLACSO-Ecuador.

Cumes, Aura (2009): "Multiculturalismo, género y feminismos: mujeres diversas, luchas complejas", enPequeño (comp.): Participación y políticas de mujeres indígenas en contextos latinoamericanos recientes, p.29-51. Quito, Ecuador:Ediciones FLACSO-Ecuador.

Espinoza, Yuderkys (2014): "Una crítica descolonial a la epistemología feminista crítica" en Revista El Cotidiano, № 184,[On Line]. Disponible en: https://es.scribd.com/document/389176220/Towards-a-Decolonial-Feminism

Forciniti, Martin y Palumbo, Mercedes (2012):"Discursos y prácticas de resistencia del feminismo indígena: desafíos para el feminismo académico y aportes para un diálogo intercultural". [on line].Disponible en: https://www.academia.edu/8753230/Discursos y pr\%C3\%A1cticas de resistencia del fem inismo ind\%C3\%ADgena desaf\%C3\%ADos para el feminismo acad\%C3\%A9mico y ap ortes para un di\%C3\%A1logo intercultural junto a Mart\%C3\%ADn Forciniti

Gargallo, Francesca (2011): "Una metodología para detectar lo que de hegemónico ha recogido el feminismo académico latinoamericano y caribeño", en Blazquez, Flores y Ríos(coords.):Investigación feminista. Epistemología, metodología y representaciones sociales, p.155-179. Ciudad de México:CEIICH/UNAM.

Gargallo, Francesca (2012): "El feminismo autónomo de las mujeres de los pueblos de AbyaYala"[on line].Disponible en:https://francescagargallo.wordpress.com/ensayos/feminismo/no-occidental/el-feminismoautonomo-de-las-mujeres-de-los-pueblos-de-abya-yala/ 
Hernández, Rosalva Aída (2008): "Feminismos poscoloniales: reflexiones desde el sur del Río Bravo", en: Suarez y Hernández (comps.):Descolonizando el Feminismo: Teorías y Prácticas desde los Márgenes,p.75-116. Madrid, España: Ediciones Cátedra.

Lorente, Maite (2005): "Diálogos entre culturas: una reflexión sobre feminismo, género, desarrollo y mujeres indígenas kiwcha" [on line]. Disponible en: https://www.ucm.es/data/cont/docs/430-2013-10-27-PP\%2001-05.pdf

Lugones, María (2008):"Towards a decolonial feminism"en Revista Hypatía, № 24, [On Line].Disponible en: https://es.scribd.com/document/389176220/Towards-a-DecolonialFeminism

Menard, André (2012):“Políticas del reducto (mapuche)”, en Actuel Marx/Intervenciones,№ 12, [On Line]. Disponible en: https://www.researchgate.net/publication/322255916 politicas del reducto mapuche

Observatorio Social (2015): Pueblos Indígenas. Síntesis de Resultados. Disponible en: http://observatorio.ministeriodesarrollosocial.gob.cl/casenmultidimensional/casen/docs/CASEN 2015 Resultados pueblos indigenas.pdf

Painemal, Millaray (2013):“Mujeres Mapuche y el aporte del feminismo en la lucha contra la violencia de género"[on line]. Disponible en:http://ftp.kaosenlared.net/kaos-tv/55844-mujeresmapuche-y-el-aporte-del-feminismo-en-la-lucha-contra-la-violencia-de-g\%C3\%A9nero

Painemal, Millaray y Álvarez, Andrea (2016):“Construyendo herramientas descolonizadas: Prevención de violencia con mujeres mapuche", en Painemal y Álvarez (comps.): Mujeres y Pueblos Originarios. Luchas y resistencias hacia la descolonización, p.72-80. Santiago de Chile: Editorial Pehuén.

Painemal, Millaray y Cañet, Isabel (2018): “¿Es que acaso debemos ser todas feministas? Reflexiones de mujeres Mapuche para un debate" [on line]. Disponible en:

http://www.mapuexpress.org/?p=23710

Quiñimil, Doris (2012): “Un proceso autoetnográfico para la descolonización feminista de las categorías mujer, mapuche, urbana, a través del aborto". Tesis (Máster Erasmus Mundus en Estudios de las Mujeres y de Género). Granada, España: Universidad de Granada, Facultad de Ciencias Sociales y Jurídicas.

Rivera, Tarcila (1999):El andar de las mujeres indígenas. Lima, Perú: Ed. Chirapac.

Segato, Rita (2011) "Género y colonialidad: en busca de claves de lectura y de un vocabulario estratégico descolonial", en: Bidaseca y Vásquez. (comps.):Feminismos y Poscolononialidad. Descolonizando el feminismo desde y en América Latina,p. 1748.Buenos Aires, Argentina: Godot. 